\title{
Preferencias en el aprendizaje de Investigación Operativa: Docencia inversa y presencial
}

\author{
María del Carmen Basa ${ }^{a}$ Marina Seguraa ${ }^{\text {, Concepción Ginestara }}{ }^{\text {, Concepción Maroto }}{ }^{a}$ \\ aDepartamento de Estadística e Investigación Operativa Aplicadas y Calidad, UPV
}

\begin{abstract}
The use of online resources for learning is increasing in business higher education, not only in the flipped classroom approach but also in order to supplement face-to-face lectures. The objectives of this paper are eliciting students' preferences and assessing the learning resources (books, slides and videos) in teaching the Operations Research in Business Administration and Management degree.

The students' preferences have been obtained using Analytic Hierarchy Process, a quantitative method, appropriate for eliciting and aggregating individual preferences to provide information from a group of people. In addition, the use and assessment of the teaching resources have been analysed using the Likert scale, a qualitative method frequently used in higher education studies.

The students who prefer videos the most are those from flipped classroom, although the videos have also affected students from traditional face-to-face teaching. In general, the slides, which synthesize and outline the concepts being studied, represent the resources more used by students of face-to-face groups. Nevertheless, there are significant differences amongst the students' preferences by group.

Finally, the videos also contribute to students skills, mainly in autonomous learning. The results and the students' suggestions encourage the teachers to continue developing additional videos with case studies and real applications.
\end{abstract}

Keywords: Student preferences, teaching resources, flipped classroom, blended learning, active learning, cooperative learning

\section{Resumen}

La creciente utilización de los recursos en línea en la formación de administración y dirección de empresas aparece tanto en el modelo de docencia inversa como complemento a la docencia presencial. Los objetivos del trabajo son obtener las preferencias de los alumnos y valorar los recursos docentes (libros, diapositivas y videos) en el aprendizaje de Investigación Operativa del Grado de Administración y Administración de Empresas. 
Las preferencias se han obtenido mediante AHP, método cuantitativo apropiado para obtener y agregar preferencias individuales, generando preferencias agregadas de grupo. Además, hemos analizado los recursos docentes mediante la escala de Likert, método cualitativo muy utilizado en los estudios de educación superior.

Los alumnos que prefieren más los videos son los de docencia inversa, aunque los videos también han influido en los estudiantes de docencia presencial. En general, las diapositivas, que sintetizan los conceptos, representan el recurso más utilizado en la docencia presencial. Sin embargo, existen diferencias significativas entre las preferencias de los alumnos por grupo.

Por último, los videos también contribuyen a la adquisición de competencias, principalmente al aprendizaje autónomo de los alumnos. Los resultados y las sugerencias recibidas nos animan a seguir desarrollando videos con casos prácticos y aplicaciones reales.

Palabras clave: Preferencias, materiales docentes, docencia inversa, aprendizaje activo, aprendizaje colaborativo

\section{Introducción}

En los últimos años, las instituciones de educación superior han experimentado diversos cambios condicionados por las exigencias de la sociedad actual. La adaptación de los títulos de grado al Espacio Europeo de Educación Superior (EEES) y el uso generalizado de las nuevas Tecnologías de la Información y Comunicación (TIC) en el ámbito de la educación superior son algunos de los factores ineludibles que han provocado un cambio en la aplicación de las metodologías docentes y una necesidad de adaptar y flexibilizar la oferta formativa a la realidad actual.

Estos nuevos cambios exigen al profesorado una renovación organizativa de las materias y una reflexión sobre cómo introducir las nuevas tecnologías, tan apreciadas por los estudiantes universitarios actuales, en sus asignaturas con el objetivo de mejorar el aprendizaje de los alumnos. Una de las tareas principales del profesorado es conseguir que los alumnos adquieran la habilidad y capacidad de seguir aprendiendo a razonar, a pensar, a deducir y a inferir conocimientos adaptándose a las nuevas metodologías de enseñanza-aprendizaje de la nueva era digital (García-Barrera, 2013).

Arbaugh et al. (2009) destacan el creciente uso de la formación online y semipresencial en los estudios de gestión y administración de empresas y defienden el interés de los métodos de investigación cuantitativos junto con los cualitativos en los estudios sobre educación. La mayoría de los trabajos publicados se han centrado más en disciplinas, tales como gestión, marketing, finanzas o contabilidad que en la formación global. En el ámbito de la Investigación Operativa, entre los trabajos recientes que analizan el impacto de los videos como material complementario a las clases presenciales podemos citar a Winch y Cahn (2015) y Sharkey y Nurre (2016).

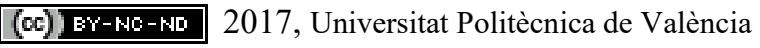


La Universitat Politècnica de València (UPV) ha desarrollado una experiencia piloto durante los cursos 2015-16 y 2016-17, basada en el modelo de clase inversa en las asignaturas de segundo y tercer curso del Grado en Administración y Dirección de Empresas (ADE). La docencia inversa (flipped classroom) consiste en invertir el modelo tradicional de docencia, sustituyendo la lección magistral por un conjunto de materiales en línea (vídeos, lecturas, artículos, etc.), gracias al apoyo de las TIC, que el alumno debe consultar de forma previa a la asistencia a las sesiones de aula. De esta forma, las sesiones de aula se convierten en clases prácticas en las que el alumno debe trabajar activamente, de forma individual o grupal, y el docente actúa como apoyo o guía (Bergmann y Sams, 2012; Zhang et al., 2012).

Entre las ventajas que ofrece el modelo de docencia inversa con respecto a la docencia presencial tradicional, podríamos destacar una mayor implicación del estudiante, un aprendizaje más significativo y colaborativo, una mayor adaptación al ritmo de cada estudiante, un mayor aprovechamiento de las horas de clase presencial y un aprendizaje más flexible y con nuevos enfoques gracias al apoyo de la tecnología (Fulton, 2012; Roehl, 2013).

Hay que destacar que los materiales empleados en este modelo son una de las claves principales para que funcione y se obtengan buenos resultados. Es por eso que se le debe dar mucha importancia a los materiales en línea de los que dispone el alumno para trabajar de forma autónoma y se deben estudiar evidencias que muestren la calidad de dichos materiales empleados para la mejora continua de la aplicación de la docencia inversa.

\section{Objetivos}

El objetivo general del presente trabajo es analizar la utilidad de los materiales elaborados para la docencia de Investigación Operativa en el aprendizaje de los alumnos de Grado en Administración y Dirección de Empresas y su contribución a la adquisición de competencias específicas y transversales.

En particular, los objetivos específicos son los siguientes:

1. Cuantificar y analizar las preferencias de los alumnos por los distintos materiales docentes desarrollados por los profesores para el aprendizaje de Investigación Operativa, que incluyen libros, transparencias y vídeos.

2. Conocer la percepción de los alumnos sobre los vídeos elaborados y su contribución al desarrollo de las competencias.

En los siguientes apartados describimos las características principales de la asignatura Investigación Operativa, la metodología docente y el sistema de evaluación que aplicamos, así como los materiales docentes desarrollados expresamente para el Grado en ADE. A continuación se detalla la metodología del trabajo, que incluye la técnica multicriterio Analytic Hierarchy Process (AHP) para elicitar las preferencias de los alumnos y la escala de Likert que habitualmente se aplica en este tipo de estudios. Por último, se presentan los resultados, las conclusiones y las propuestas de mejora. 


\section{Desarrollo de la innovación}

\subsection{Descripción de la asignatura, metodología docente y sistema de evaluación}

La Investigación Operativa forma parte de la materia básica y obligatoria "Métodos cuantitativos para la empresa" y se imparte en el primer cuatrimestre del tercer curso del Grado en ADE (Facultad de Administración y Dirección de Empresas, UPV). Su objetivo es que los alumnos aprendan a formular modelos de optimización para resolver problemas reales de toma de decisiones, así como los conceptos y las técnicas necesarias para resolver modelos de programación lineal, entera, no lineal, multiobjetivo y multicriterio mediante software profesional e interpretar los resultados con la finalidad de mejorar las decisiones empresariales.

Esta asignatura tiene 6 créditos ECTS, 3 créditos de teoría y 3 créditos de prácticas, 1 de prácticas de aula y 2 de prácticas informáticas. En el aula se combina la presentación de modelos, métodos y aplicaciones por parte del profesor con la realización de ejercicios por parte de los alumnos, generalmente en grupos, aplicando el aprendizaje colaborativo. También, en las diez sesiones de dos horas en el aula informática los alumnos trabajan en grupos formulando modelos, que después resuelven e interpretan las soluciones, con la ayuda del profesor. La implantación de esta metodología, enfocada hacia el aprendizaje de los alumnos, tiene ya una larga trayectoria y su origen en varios Planes de Innovación Educativa desarrollados en la UPV (Maroto et al., 1997; Alcaraz et al., 2003; Alcaraz et al., 2004).

La Investigación Operativa se organiza en tres grupos: mañanas, tardes y grupo ARA (Alto Rendimiento Académico). En este último grupo la asignatura se imparte en inglés. Además, durante los cursos 2015-16 y 2016-17 se ha implantado un cuarto grupo de docencia inversa en el marco de una experiencia piloto. El grupo de docencia inversa tiene las mismas clases de laboratorio informático que los demás, pero menos horas de aula, ya que en este modelo el trabajo previo de los alumnos sustituye la presentación del profesor, enfocándose las clases de aula como clases prácticas.

El sistema de evaluación es el mismo para todos los grupos, basado en la evaluación continua del trabajo realizado en grupos de 2-3 alumnos en diez sesiones de prácticas informáticas. En estas clases se plantean y resuelven modelos de toma de decisiones y se interpretan los resultados. Al finalizar cada clase práctica los alumnos deben subir el modelo y el informe realizado a poliformaT. Estos informes representan el 20\% de la calificación final. Asimismo se realizan 2 exámenes, uno sobre la primera mitad del temario y el otro sobre la segunda mitad, cada uno de los cuales vale el $40 \%$ de la calificación final. Por tanto, los alumnos tienen a mitad de semestre una calificación parcial (P1) correspondiente al 50\% de la nota global y otra P2 de la segunda parte de la materia. Los alumnos que no superan la asignatura pueden realizar un examen de recuperación de todo el temario ( $80 \%$ de la calificación), manteniéndose la nota de las prácticas informáticas que se haya obtenido en el curso.

(cc) EY-NC-ND 2017, Universitat Politècnica de València 


\subsection{Diseño del material docente}

Antes de la implantación del grupo de docencia inversa el material docente básico de la asignatura estaba formado por un libro de texto expresamente diseñado para impartir la asignatura en el grado en ADE y transparencias de apoyo a las presentaciones del profesor. El libro incluye tanto el material para las explicaciones teóricas como los casos prácticos a desarrollar y resolver en el aula y en el laboratorio informático. Este libro está publicado en castellano y en inglés para el grupo ARA y ambos disponibles en papel y como libros electrónicos (Maroto et al., 2012, Maroto et al., 2014).

Las transparencias en Powerpoint de los 8 temas de la asignatura, basadas en los libros citados, están disponibles en poliformaT para los alumnos, también en castellano e inglés. Durante el curso 2015-16 se grabaron vídeos con la explicación de las transparencias de toda la asignatura como apoyo adicional para los alumnos del grupo de docencia inversa. Estos vídeos se grabaron mediante la herramienta Powerpoint y están disponibles en PoliformaT con enlaces al repositorio UPV \{Media\} (https://media.upv.es). Los alumnos tienen disponible un total de 17 vídeos de toda la asignatura con una duración entre 8 min y 55 min, dependiendo de la complejidad de los temas y del número de ejemplos.

Aunque se prepararon para el grupo de docencia inversa, los vídeos están disponibles para los alumnos de todos los grupos. Conscientes de la importante contribución que puede suponer la disponibilidad de vídeos como material complementario a las clases presenciales, durante el curso 2016-17 se han preparado tres vídeos en inglés para el grupo ARA en los que se explican conocimientos difíciles para algunos alumnos, pero básicos y muy importantes para el aprendizaje de la materia.

\subsection{Metodología}

Para alcanzar los objetivos planteados en este estudio se ha diseñado una encuesta con el fin conocer las preferencias y la percepción de los alumnos sobre el material docente. La encuesta se ha dirigido a todos los grupos de la asignatura Investigación Operativa del grado en ADE durante el curso académico 2016-2017.

El cuestionario se entregó en papel a los alumnos en clase en la última semana del curso (diciembre 2016) y también se envió a través de un formulario on-line, elaborado con la herramienta Google Docs, a todos los alumnos para que pudieran responder aquellos que no hubieran estado presentes en el aula el día que se realizó la encuesta. La encuesta es anónima en ambos casos.

El cuestionario se ha dividido en cuatro partes:

Parte I. Características del alumno (grupo, asistencia habitual a las clases, calificación obtenida en la primera parte de la asignatura que representa el 50\% de la evaluación total, Parcial 1). 
Parte II. Preferencias y valoración de los materiales docentes de la asignatura

II.a) Preferencias por los materiales docentes para el aprendizaje de Investigación Operativa mediante el método AHP.

II.b) Grado de utilización del material docente (libro, transparencia y vídeos). Se ha empleado una escala de Likert de 5 puntos ( $1=$ Nada, $5=$ Mucho).

II.c) Valoración de los vídeos como material de apoyo. Se ha empleado una escala de Likert de 5 puntos (1=Nada, $5=$ Mucho).

Parte III. Contribución de los vídeos al desarrollo de las competencias específicas (CE) y transversales $(\mathrm{CT})$ trabajadas en la asignatura. Se ha empleado una escala de Likert de 5 puntos ( $1=$ Ninguna, $5=$ Muy Alta $)$.

Parte IV. Sugerencias de mejora para la elaboración de materiales docentes.

Para obtener las preferencias de los alumnos por los materiales para el aprendizaje de la Investigación Operativa se ha diseñado una pregunta basada en el método AHP que nos permite determinar el peso de cada tipo de material, tanto para los alumnos individualmente como a nivel de grupo.

La figura 1 ilustra la jerarquía del método AHP, ampliamente implantado para elicitar las preferencias de los stakeholders en numerosos ámbitos de investigación (Saaty y Peniwati, 2008). En la asignatura se había explicado previamente este método como técnica de toma de decisiones colaborativa. En síntesis, teniendo en cuenta que el objetivo global es el aprendizaje de Investigación Operativa los alumnos comparan dos a dos los distintos tipos de materiales: libro, transparencias y vídeos, asignando su importancia relativa mediante la escala cuantitativa de Saaty en una matriz de comparaciones (Saaty y Vargas, 2001).

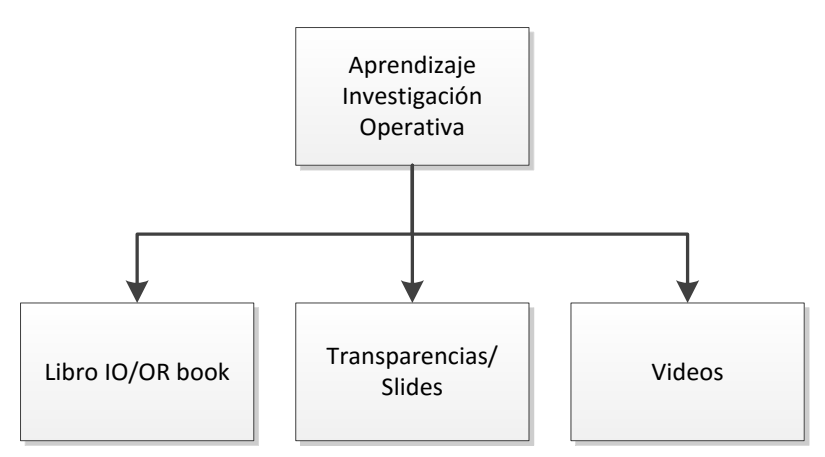

Fig. 1 Jerarquía de los materiales docentes en la asignatura Investigación Operativa 


\section{Resultados}

\subsection{Porcentaje de respuestas y representatividad}

La tabla 1 recoge el número de alumnos matriculados en cada grupo y la respuestas obtenidas. Se ha obtenido una tasa de respuesta del $58,8 \%$ del total, si bien este porcentaje es menor en el grupo de docencia inversa, que presenta un número muy reducido de alumnos matriculados, similar al de otras asignaturas del proyecto piloto. Además dos de los ocho alumnos tenían exención de asistencia y no realizaron ninguna actividad a lo largo del curso ni se presentaron a los exámenes. Teniendo en cuenta lo anterior, la tasa de respuesta real sería similar a la del grupo más numeroso de mañanas.

Tabla 1. Número de alumnos matriculados y porcentaje de respuesta por grupos

\begin{tabular}{|c|c|c|c|}
\hline Grupo & Matriculados & Respuesta & \% Respuesta \\
\hline Mañanas & 64 & 32 & $50 \%$ \\
\hline Tardes & 31 & 23 & $74,19 \%$ \\
\hline ARA & 33 & 22 & $66,6 \%$ \\
\hline D. Inversa & 8 & 3 & $37,5 \%$ \\
\hline TOTAL & 136 & 80 & $58,8 \%$ \\
\hline
\end{tabular}

De los 80 alumnos que responden, 74 cumplimentaron la encuesta presencialmente en clase y 6 la remitieron online, 3 de los cuales no asisten a clase y los otros 3 sí. Casi la totalidad de alumnos que responden la encuesta asisten habitualmente a las clases de teoría y prácticas de aula $(92,50 \%)$. Hay que destacar que asistir a clase de forma habitual para el grupo de docencia inversa se refiere a asistir a las clases presenciales, que representan menos horas en este caso que en los restantes grupos. Este nivel de representatividad se tendrá en cuenta a la hora de interpretar los resultados.

Como se puede observar en la figura 2 los alumnos que responden la encuesta, que asisten mayoritariamente a clase, presentan mejores calificaciones con mayor porcentaje de buenas notas, en especial sobresalientes, y con menor tasa de suspensos en comparación con el total de alumnos. Se utiliza la nota Parcial 1 debido a que es la única disponible en el momento de cumplimentar el cuestionario y con la finalidad de comparar la tasa de rendimiento y las calificaciones de los alumnos que responden frente al total. 


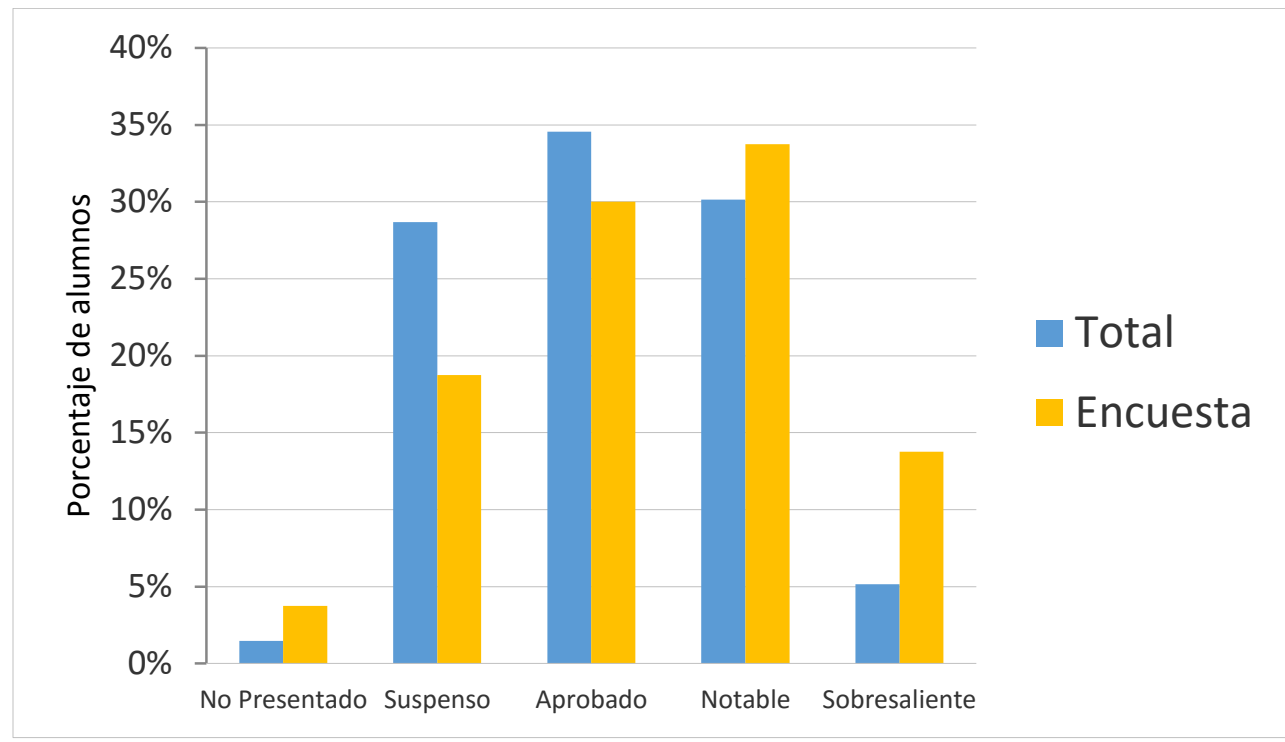

Fig. 2 Porcentaje de alumnos del curso y de los que responden la encuesta según Nota Parcial 1

\subsection{Preferencias de los alumnos por los diferentes tipos de materiales docentes}

Previo al cálculo de las preferencias mediante AHP hay que determinar el índice de inconsistencia de la matriz de comparación por pares de cada alumno. El 32,5\% del total presenta una inconsistencia elevada (superior a 0,12 ) y por tanto estas respuestas se eliminan del análisis. Este porcentaje varía entre el $27,3 \%$ en el grupo de tardes y el $36,4 \%$ en el grupo ARA. Aunque sólo 6 de los 80 alumnos que han respondido la encuesta no asisten habitualmente a clase, el $66 \%$ de sus respuestas son inconsistentes, duplicando el porcentaje de inconsistencia de los estudiantes que sí asisten a las clases, siendo éste un indicador de un mayor grado de aprendizaje de los alumnos que asisten a clase, ya que todos habían realizado un caso práctico en el laboratorio basado en esta técnica. Hay que resaltar que el nivel de asistencia a las prácticas de laboratorio informático, base de la evaluación continua, está cercano al $100 \%$.

El conocimiento de la técnica AHP de los alumnos que asisten a clase y su repercusión en la capacidad de responder a las comparaciones por pares de forma más consistente también se confirma al comparar el porcentaje de alumnos cuyas matrices han presentado una consistencia aceptable con otra investigación en la que participaron alumnos de ADE sin estudio previo de la técnica. En este caso el porcentaje de matrices inconsistentes fue del 57,5 (Segura, 2011).

La figura 3 representa las preferencias de los alumnos por el libro, las transparencias y los vídeos en el aprendizaje de Investigación Operativa. Los pesos a nivel de grupo y total se han obtenido aplicando la media geométrica a las respuestas de los alumnos consistentes para obtener una matriz agregada, que es consistente y a partir de la cual se calculan los pesos de los materiales para cada grupo, tal y como se procede en toma de decisiones colaborativa 
para construir la preferencia de un grupo de personas a partir de las preferencias individuales (Saaty y Peniwati, 2008).

Aunque a nivel global las transparencias son el recurso más utilizado por la mayoría, la figura 3 muestra diferencias importantes por grupos. El grupo de mañanas es el que más peso da a las transparencias, mientras que los vídeos son mucho más importantes en docencia inversa. Por otra parte, el grupo con docencia en inglés es el que más peso da al libro, con un peso similar a las transparencias y el grupo de tardes destaca por ser el grupo presencial que más peso da a los vídeos. Estas diferencias entre grupos (sin considerar la docencia inversa por el reducido número de alumnos, por lo que $\mathrm{N}=77$ ) son significativas estadísticamente al $95 \%$ aplicando el test de Kruskal-Wallis en todos los casos, excepto la comparación entre videos y libro cuya diferencia es significativa al $90 \%$.

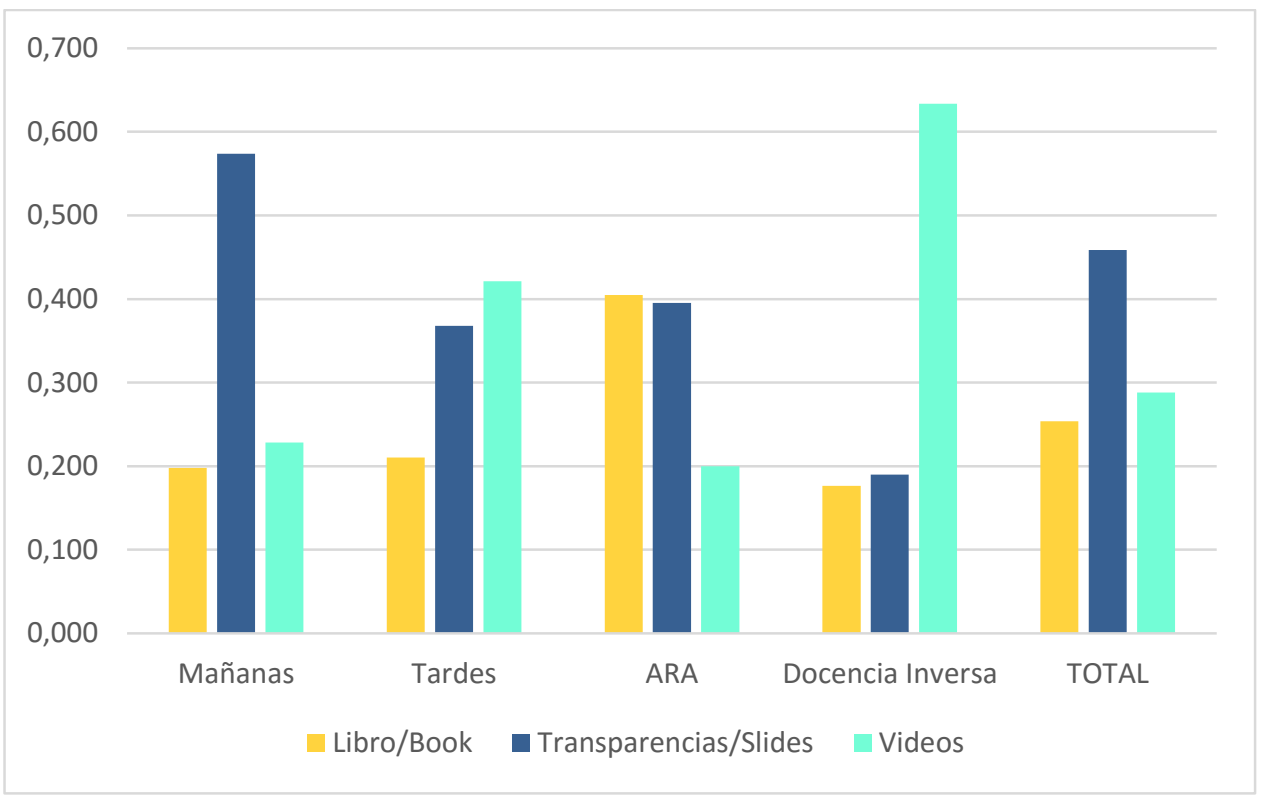

Fig. 3 Preferencias de los alumnos por los materiales docentes. Peso obtenido mediante AHP.

\subsection{Grado de utilización del material docente de la asignatura}

La figura 4 muestra el grado de utilización del material docente, que corresponde al libro diseñado expresamente para la asignatura, las transparencias y los vídeos grabados. El grado de utilización del material se ha analizado según la asistencia o no a clase con el fin de identificar las posibles diferencias entre los dos grupos, aunque hay que tener en cuenta que la escasa representatividad de los que no asisten a clase, ya que sólo hay 6 respuestas frente a 74 que sí asisten habitualmente.

Los resultados muestran que los vídeos son el material docente más utilizado por los alumnos que no asisten $(4,83)$, seguido de las transparencias (3) y el libro $(2,83)$. Por el contrario, los 
alumnos que asisten habitualmente a clase valoran con un grado de utilización medio las transparencias $(3,84)$, seguidas del libro $(2,8)$ y finalmente los vídeos $(2,61)$, aunque hay que destacar que no hay mucha diferencia entre la puntuación media entre libro y vídeos para los alumnos que asisten a clase.

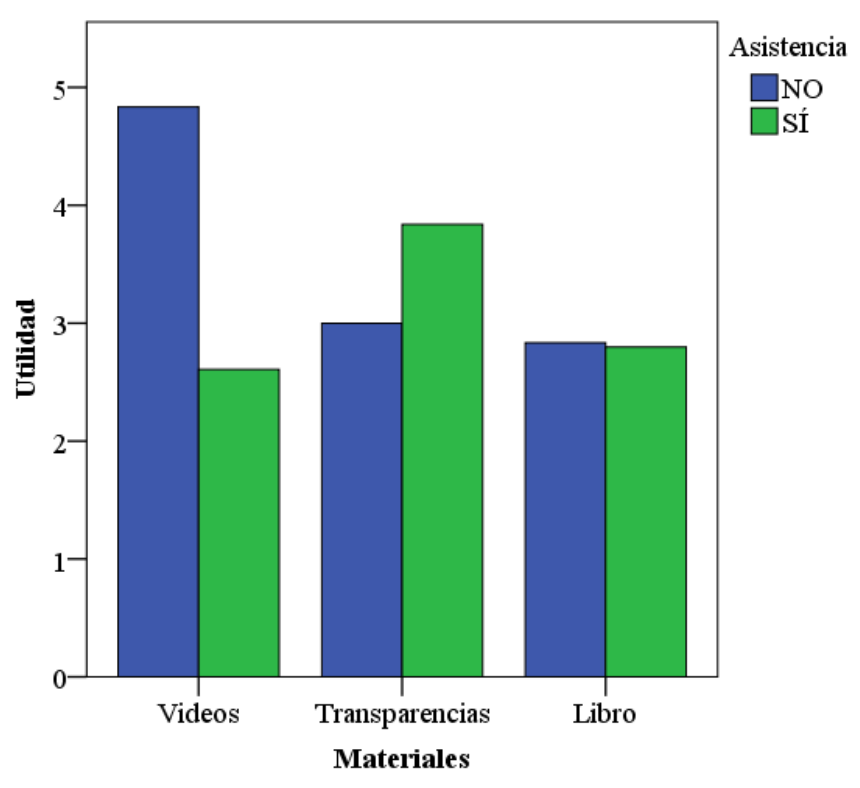

Fig. 4 Utilización material docente según asistencia a clase basado en escala de Likert

La figura 5 representa el grado utilización material docente según la calificación obtenida. Se observa que en promedio las transparencias son el material de aprendizaje más utilizado por los alumnos, independiente del mayor o menor aprovechamiento reflejado en la calificación obtenida a mitad de curso. Lo mismo se puede decir del grado de utilización del libro de texto. Sin embargo, la utilización de los vídeos es mayor en aquellos alumnos que tienen calificaciones más bajas (suspenso y aprobado). El número de alumnos no presentados es muy reducido, tanto a nivel global de la asignatura $(4,3 \%)$ como en los que responden la encuesta $(3,8 \%)$. Esta mayor proporción de alumnos suspendidos entre los que más ven los videos, unida a que son también los que no asisten a las clases presenciales teóricas y prácticas en aula, consideramos que es debida a que estos alumnos no trabajan ni resuelven los casos prácticos, muy relevantes para asimilar los conceptos en asignaturas cuantitativas como Investigación Operativa. 


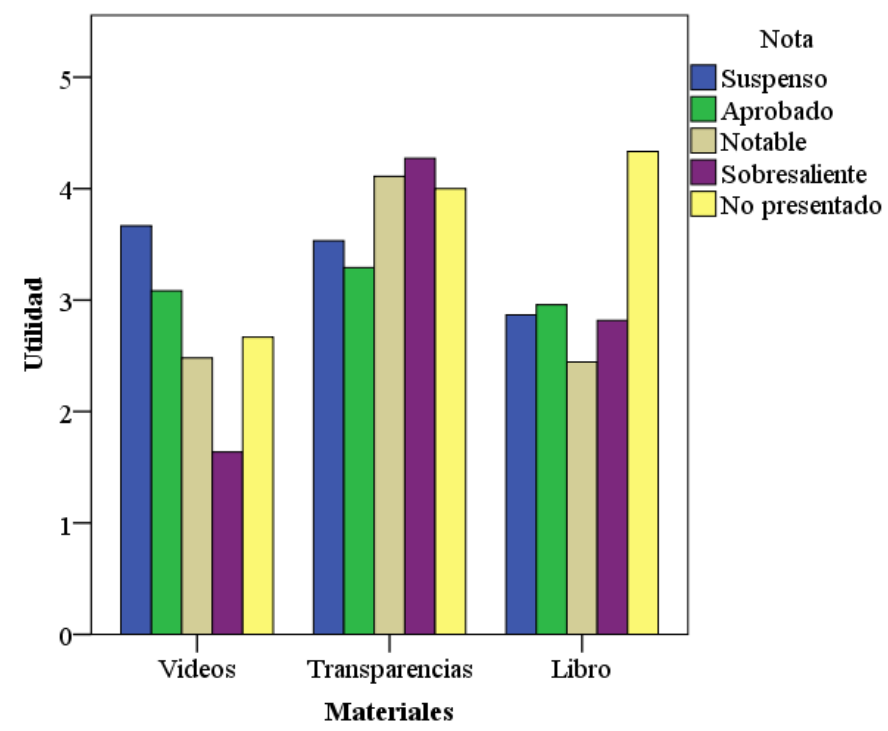

Fig. 5 Grado utilización material docente según la calificación de los alumnos

En la figura 6 podemos observar la distribución del grado utilización del material docente por grupos basado en la escala de Likert. Al comparar estos resultados con los obtenidos mediante AHP (figura 3) vemos cómo la escala de Likert discrimina entre grupos mucho menos que la técnica AHP, basada en una escala cuantitativa ( Cochran, 2012).

Las diferencias más importantes se observan en los resultados de ambas metodologías, como la mayor utilización de las transparencias por el grupo de mañanas y de vídeos por el grupo de docencia inversa. Si bien a partir de AHP, las diferencias entre grupos son todas significativas, no ocurre lo mismo al aplicar el test no paramétrico de Kruskal-Wallis con una corrección de Bonferroni para comparaciones múltiples a los datos de la encuesta procedentes de la escala de Likert. En este análisis no se ha considerado el grupo de docencia inversa por el pequeño número de alumnos de esta modalidad.

Los resultados obtenidos muestran que no existen diferencias significativas entre los grupos de mañana, tarde, y ARA para el grado de utilización de los vídeos. Por el contrario, sí que existen diferencias significativas al 95\% de confianza entre los grupos para el grado de utilidad de las transparencias. Estas diferencias se observan entre el grupo de la mañana y el grupo de la tarde, siendo el grupo de la mañana el que más emplea las transparencias como material docente. Finalmente, el grado de utilidad del libro también es estadísticamente significativo al $95 \%$ para los distintos grupos, encontrándose dichas diferencias entre el grupo ARA y el de la mañana, siendo el grupo ARA el que emplea más el libro como material docente de la asignatura. 


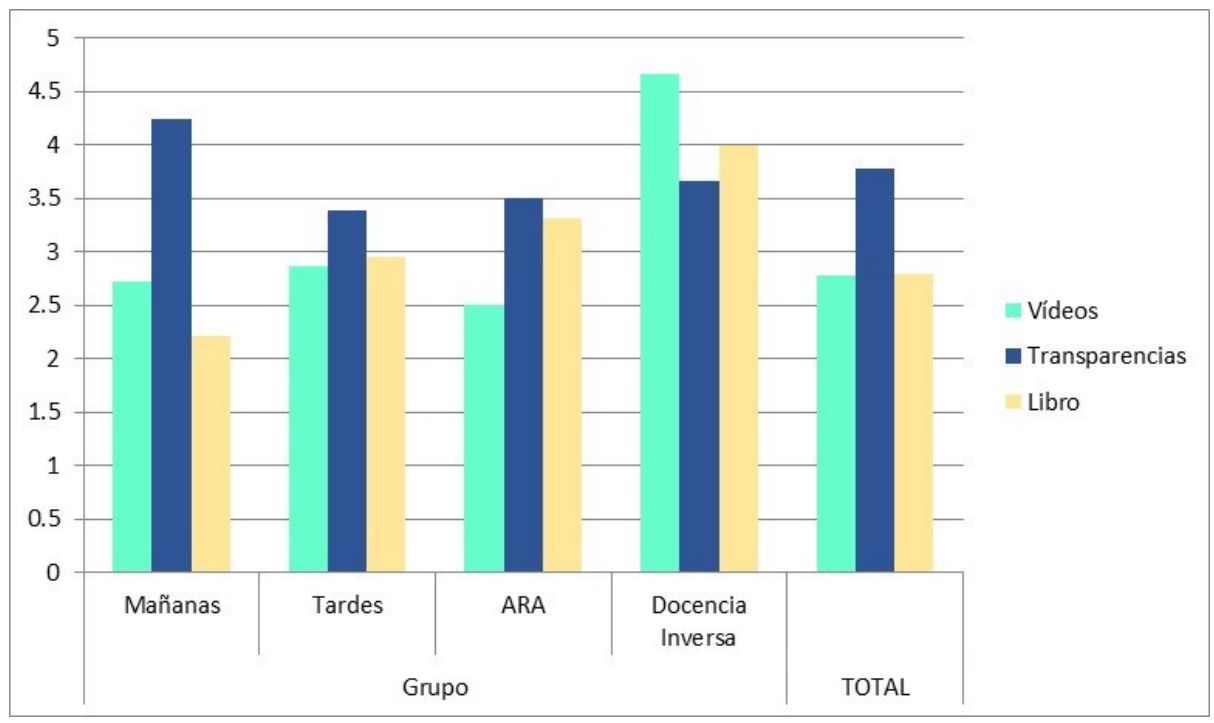

Fig. 6 Grado utilización material docente por grupos según la escala de Likert

\subsection{Valoración de los vídeos como material de apoyo}

A continuación se presentan los resultados sobre la utilidad, calidad y cantidad de los vídeos como material de apoyo de la asignatura (tabla 2) usando la escala de Likert. Los alumnos han asignado una puntación de 3,90 a la calidad de los vídeos, un 3,53 a la utilidad que les dan y una puntuación menor $(3,35)$ a la cantidad de estos. Se puede interpretar que los alumnos desearían un mayor número de vídeos. La tabla 2 pone de manifiesto que los alumnos del grupo de docencia inversa son los que mejor evalúan los vídeos tanto en utilidad, cantidad y calidad.

Tabla 2. Valoración de las características de los vídeos

\begin{tabular}{|c|c|c|c|c|}
\hline \multicolumn{1}{|c|}{} & Utilidad & Cantidad & Calidad \\
\hline \multirow{4}{*}{ Grupo } & Mañanas & 3,74 & 3,96 & 4,30 \\
\cline { 2 - 5 } & Tardes & 3,15 & 2,85 & 3,35 \\
\cline { 2 - 5 } & ARA & 3,50 & 2,79 & 3,86 \\
\cline { 2 - 5 } & Docencia Inversa & 4,67 & 4,67 & 4,67 \\
\hline \multicolumn{2}{|c|}{ TOTAL } & 3,53 & 3,35 & 3,90 \\
\hline
\end{tabular}

\subsection{Contribución de los vídeos al desarrollo de competencias}

Según el estudio de competencias, los alumnos opinan que los vídeos tienen una mayor contribución a la competencia específica "Aprender autónomamente", que es uno de los objetivos de los vídeos, el trabajo autónomo por parte de los alumnos. A esta competencia,

(cc) EY-NC-ND 2017, Universitat Politècnica de València 
le siguen en puntuación las competencias transversales "Aplicación y pensamiento práctico" y "Análisis y resolución de problemas".

Por el contrario, la competencia menos valorada es el "Trabajo en equipo y liderazgo", ya que en este caso, los vídeos son una herramienta muy útil para el trabajo autónomo, pero, según los alumnos no fomenta el trabajo en equipo. Este resultado contrasta con los mencionados en otros trabajos publicados (Arbaugh et al., 2009). Hay que tener en cuenta que esta competencia se trabaja en las clases prácticas de laboratorio informático y en las clases prácticas de aula donde los alumnos trabajan en grupos y aprenden de forma colaborativa.

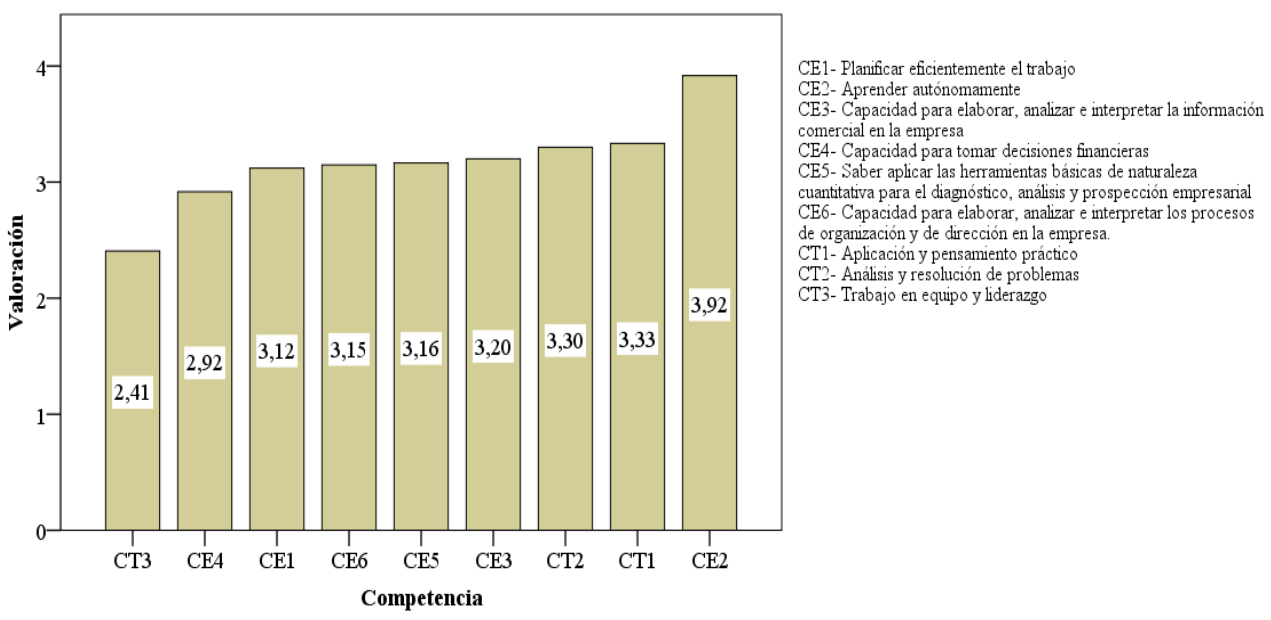

Fig. 7 Contribución de los vídeos al desarrollo de competencias

\subsection{Sugerencias de los alumnos para la mejora del material docente}

El cuestionario incluye una pregunta abierta para recoger las sugerencias de mejora y observaciones que han realizado los alumnos sobre el material docente de la asignatura.

En primer lugar, un número muy importante de alumnos destaca los exámenes resueltos, disponibles en PoliformaT, como un material importante para preparar la asignatura. Grabar videos con los ejercicios que se hacen en las clases presenciales y la solución de los exámenes es otra de las sugerencias. Asimismo, sugieren reducir la duración de los videos, incluir más ejemplos de aplicaciones reales y más explicaciones escritas de los gráficos y esquemas en las transparencias. Algunos alumnos de los grupos presenciales indican que no conocían la existencia de vídeos destinados a la docencia inversa. 


\section{Conclusiones}

La principal contribución de este trabajo es la aplicación de un método cuantitativo muy utilizado en toma de decisiones colaborativa (AHP), poniendo de manifiesto su mayor poder discriminante frente a otros métodos basados en escalas cualitativas, de uso más frecuente en estudios sobre educación. Autores como Arbaugh et al. (2009) y Cochran (2012) defienden el interés y complementariedad de ambos tipos de metodologías.

Si bien los alumnos del grupo de docencia inversa son los que más prefieren, utilizan y valoran los vídeos como material de aprendizaje, éstos también han tenido una influencia importante en los alumnos de docencia presencial.

Globalmente las transparencias, que recogen de forma sintética y esquemática los conceptos de la asignatura, son el recurso de aprendizaje más utilizado por los alumnos de docencia presencial, independientemente de su calificación. El libro de texto se utiliza más en el grupo ARA y el grupo de tardes recoge los alumnos presenciales que más utilizan los vídeos.

Los vídeos, grabados expresamente para la docencia inversa, amplían y flexibilizan las oportunidades de aprendizaje y permiten mayor adaptación al ritmo de estudio de cada alumno que la clase presencial. Por tanto, no sólo ayudan a aquellos que no han asistido a alguna clase, sino también a aquellos que tienen mayor dificultad con la materia, que pueden pararlos, tomar notas sobre las transparencias o verlos varias veces, si es necesario tal y como se ha puesto de manifiesto también en otros trabajos (Sharkey y Nurre, 2016).

La relación positiva que hemos encontrado entre la visualización de vídeos y los alumnos que no han aprobado el primer parcial se puede atribuir a que en los vídeos no se han incluido casos prácticos adicionales resueltos, que sí se trabajan en el aula y que los alumnos que no asisten a clase y la sustituyen por la visualización de vídeos únicamente, no los resuelven de forma autónoma. El trabajo de estos casos prácticos es esencial para la comprensión de los conceptos en materias de naturaleza cuantitativa como es el caso de Investigación Operativa.

En cuanto a la percepción de los alumnos sobre los vídeos y su contribución al desarrollo de las competencias de la asignatura se confirma la importante contribución de los vídeos en la competencia de aprender autónomamente, que es la que recibe la mayor valoración. Por el contrario, el uso de vídeos no contribuye en gran medida al desarrollo de la capacidad 'trabajo en equipo', competencia que se trabaja en las clases prácticas de aula y laboratorio.

El hecho de que los alumnos tengan los exámenes resueltos con explicaciones detalladas, por una parte mejora el aprendizaje de los alumnos y por otra, reduce su necesidad de tutorías. También se ha observado que la disponibilidad de vídeos para la docencia inversa, con la presentación de los contenidos de las clases presenciales, ha reducido la asistencia a clase de los alumnos, en particular en el grupo más numeroso.

Los resultados del trabajo, unido a las sugerencias de los alumnos, nos indican que el aprendizaje se podría mejorar completando el material con vídeos sobre una amplia variedad de casos prácticos y aplicaciones reales. En esta línea continuaremos intentado mejorar y facilitar el aprendizaje de Investigación Operativa.

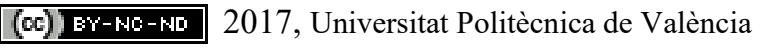




\section{Referencias}

ALCARAZ, J. et al. (2003). "Autonomous web-based learning of Operations Research" en International Conference on Engineering Education ICEE2003. Abstracts. Valencia.

ALCARAZ, J. et al. (2004). "Teaching Innovation of Operations Research Through a Web-Based System" en 7th Internacional Workshop on Higher Education and its Perspectives. Actas. Havana (Cuba).

ARBAUGH, J.B. et al. (2009). "Research in online and blended learning in the business disciplines: Key findings and possible future directions" en Internet and Higher Education 12, 71-87.

BERGMANN, J. y SAMS, A. (2012). Flip YOUR Classroom. Reach Every Student in Every Class Every Day. International Society for Technology in Education.

COCHRAN, J.J. (2012). "You want them to remember? Then make it memorable! Means for enhancing operations research education" en European Journal of Operational Research 219, 659-670.

GARCÍA-BARRERA, A. (2013). "El aula inversa: cambiando la respuesta a las necesidades de los estudiantes” en Avances en Supervisión Educativa, 19.

FULTON, K. (2012). "Upside down and inside out: Flip your classroom to improve student learning" en Learning \& Leading with Technology, 39, p. 12-17.

MAROTO, C. et al. (2012). Investigación Operativa en Administración y Dirección de Empresas. Valencia: Editorial UPV. Disponible en papel y libro electrónico.

MAROTO, C. et al. (2014). Operations Research in Business Administration and Management. Valencia: Editorial UPV. Disponible en papel y libro electrónico.

MAROTO, C. et al. (1997). "Operational Research Teaching in Computer Science through Six Years of Innovative Education Experience" en XV EURO and XXXIV INFORMS Joint International Meeting: OR/MS for the New Millennium. Abstracts.

ROEHL, A. (2013). "Bridging the field trip gap: Integrating webbased video as teaching and learning partner in interior design education" en Journal of Family \& Consumer Sciences, 105, p. 42-46.

SAATY, T.L. y PENIWATI, K. (2008). Group decision making: drawing out and reconciling differences. RWS Publications.

SAATY, T.L. y VARGAS, L.G. (2001). Models, methods, concepts \& applications of the analytic hierarchy process. Kluwer Academic Publishers.

SEGURA, M. (2011). Gestión forestal sostenible y participativa del monte mediterraneo: agregación de preferencias mediante AHP y programación por metas. Trabajo Fin de Master. Master Universitario de Ingenieria de Análisis de Datos, Mejora de Procesos y Toma de Decisiones. Universitat Politecnica de Valencia.

SHARKEY, T.C. y NURRE, S.G. (2016). "Video Tutorials Within an Undergraduate Operations Research Course: Student Perception on Their Integration and Creating A Blended Learning Environment" en INFORMS Transactions on Education 17(1):1-12.

WINCH, J.K y CAHN, E.S. (2015). “Improving Student Performance in a Management Science Course With Supplemental Tutorial Videos" en Journal of Education for Business, 90:7, 402-409.

ZHANG, J., WANG, Y. y ZHANG, B. (2012). "Introducing a New Teaching Model: Flipped Classroom" en Journal of Distance Education, vol 4, p. 46-51. 